\title{
Veranstaltungen
}

http://doi.org/10.1515/bd-2019-0054

\section{ASpB-Tagung 2019: „Hoch hinaus! Innovation in Spezialbibliotheken auf bewährtem Fundament"“}

Die 37. Fachtagung der Arbeitsgemeinschaft der Spezialbibliotheken e.V. (ASpB) findet in Kooperation mit der Universitätsbibliothek Johann Christian Senckenberg der Johann Wolfgang Goethe-Universität Frankfurt vom 4.-6. September 2019 in Frankfurt am Main statt.

Ist Ihre Bibliothek smart oder möchte es gerne werden? Digitalisieren Sie eigene Bestände oder realisieren Digitalisierungsprojekte im Verbund? Beteiligen Sie sich an Fachinformationsdiensten oder an den Initiativen zum Aufbau einer Nationalen Forschungsdateninfrastruktur? Wie entwickeln sich die Aufgabenprofile von Firmenbibliotheken? Und wie verfahren Sie in der Bewahrung, Erschließung und Vermittlung von nicht-textlichen Sammlungen, z. B. Filmen, Bildern oder Objekten? Diesen und weiteren Fragen möchten wir mit Ihnen in Frankfurt nachgehen sowie Anregungen und Best-Practice-Beispiele liefern, die unmittelbar in Ihrem Arbeitsalltag umsetzbar sind.

Die Tagung wird sich mit folgenden inhaltlichen Schwerpunkten beschäftigen:

- Themenbereich 1: „Die neue Altstadt“ - Smarte und fluide Bibliothekskonzepte

- Themenbereich 2: „Business Class“ - Neue Profile für Firmenbibliotheken

- Themenbereich 3: „(Inter)nationales Drehkreuz“ - FID, NFDI \& Co.: Spezialbibliotheken in übergreifenden Infrastrukturen

- Themenbereich 4: „Sieben Kräuter, aber welche?“ - Nicht-textliche Sammlungen, Mediendatenbanken, Spezialbestände und ihre Sichtbarkeit

- Themenbereich 5: „Grüne Soße, Grüner Weg?“ - Fragen und Anwendungen von Open Access

- Themenbereich 6: „Oberfrank und Unterfurt“ - Rund um die Erschließung: Fachthesauri und maßgeschneiderte Klassifikationen

- Themenbereich 7: Goethe goes Cloud“ - Digitalisierungsprojekte: Möglichkeiten für kleine Einrichtungen 
Darüber hinaus wird es eine Postersession geben, bei der die besten Projektideen mit einem Preisgeld auf der Tagung präsentiert werden. Hierfür können bis zum 31. Juli Vorschläge eingereicht werden.

Freuen Sie sich schon jetzt auf ein informatives, praxisbezogenes Programm, eine umfangreiche Fachausstellung und ein vielfältiges Rahmenprogramm. Weitere Informationen zum Programm, zu Registrierung und zum Veranstaltungsort finden sich auf der Tagungswebsite: www.aspbtagung.de.

\section{Kontakt:}

Kerstin Schoof

Vorsitzende des Vorstands der Arbeitsgemeinschaft der Spezialbibliotheken (ASpB)

Leiterin der Bibliothek, Max-Planck-Institut für empirische Ästhetik Grüneburgweg 14, 60322 Frankfurt

Tel.: 069 / 698300 479 640, E-Mail: kerstin.schoof@aesthetics.mpg.de, http://www.aesthetics.mpg.de, http://www.aspb.de/de 